\title{
Abnormal sweat electrolytes in symptomatic human immunodeficiency virus infection in a child
}

\author{
C H SKEOCH, N A COUTTS, K M GOEL, E A FOLLETT
}

\begin{abstract}
A $3 \frac{1}{2}$ year old girl presented with failure to thrive and a five month history of diarrhoea and recurrent cough. The results of sweat sodium tests suggested a diagnosis of cystic fibrosis; but atypical organisms were found (Haemophilus influenzae, Candida albicans, but no Staphylococcus aureus), she failed to respond to treatment, and her sweat sodium concentrations fell in response to fludrocortisone. She also had hyperglobulinaemia, neutropenia, and reduced numbers of T4 lymphocytes, which prompted the performance of a test for antibody to human immunodeficiency virus (HIV). This proved positive, and she was treated with co-trimoxazole, zidovudine, and human immunoglobulin. Both parents and two siblings were also positive for HIV, though all had normal sweat sodium concentrations.
\end{abstract}

Children with symptoms suggestive of cystic fibrosis but who also show atypical features, as in this case, should have their HIV state checked.

\section{Introduction}

The diagnosis of cystic fibrosis in a child often depends on the clinical features of failure to thrive and recurrent respiratory infections along with the laboratory findings of abnormal sweat electrolyte concentrations. We have recently assessed a child with such clinical and laboratory findings who had a final diagnosis of symptomatic human immunodeficiency virus (HIV) infection.

\section{Case report}

A $31 / 2$ year old girl presented with a five month history of diarrhoea, recurrent cough, and failure to thrive. She was the second of four children whose ages ranged from 4 months to 6 years. The parents were nonconsanguinlous and the mother had received a blood transfusion after the birth of the eldest child. The child herself had never received blood or blood products.

Our patient was admitted to hospital because of exacerbation of her cough. During the three months before diagnosis she had had several episodes of fever and cough and showed both clinical and radiological changes of bronchopneumonia. She was also failing to thrive, her weight falling from the 50th centile at the age of $1 \frac{1}{2}$ years to less than the third centile at the time of the initial assessment.

Bacteriological investigation showed her sputum to be growing Haemophilus influenzae and Candida albicans. She was treated with appropriate antibiotics, and in view of the clinical picture sweat tests were performed. Sweat sodium concentration was $59 \mathrm{mmo} / \mathrm{kg}$ and $43 \mathrm{mmol} / \mathrm{kg}$ on two consecutive tests with adequate weight of sweat.

Royal Hospital for Sick Children, Yorkhill, Glasgow G3 8S]

C H SKEOCH, MB, MRCP, senior registrar in medical paediatrics

K M GOEL, MD, FRCP, consultant paediatrician

Inverclyde Royal Hospital, Greenock

N A COUTTS, MB, FRCP, consultant paediatrician

Ruchill Hospital, Glasgow

E A FOLLETT, PHD, MRCPATH, top grade virologist

Correspondence to: Dr Skeoch.
Her IgG, IgA, and IgM concentrations were all raised $(290 \mathrm{IU} / \mathrm{ml}$, $400 \mathrm{IU} / \mathrm{ml}, 600 \mathrm{IU} / \mathrm{ml}$ respectively.) She had a fluctuating white cell count and on several occasions had neutropenia, the lowest total white blood cell count being $2.9 \times 10^{9} / 1$ with $1 \cdot 1 \times 10^{9} / 1$ neutrophils. The haemoglobin remained around $80 \mathrm{~g} / 1$ and the platelet count was normal.

A repeat sweat sodium concentration was $108 \mathrm{mmol} / \mathrm{kg}$, with a chloride concentration of $93 \mathrm{mmol} / \mathrm{kg}$ and weight of sweat of $156 \mathrm{mg}$. A further sample produced similar results. The child was started on pancreatic replacement therapy and given intensive physiotherapy and antibiotic treatment while awaiting the results of tests of polymorphonuclear leucocyte and $T$ cell function. With this treatment there was no clinical improvement.

Fludrocortisone was then given orally for two days in a dose of $2 \mathrm{mg} / \mathrm{m}^{2}$ per day. A further sweat test showed a sweat sodium concentration of $31 \mathrm{mmo} / \mathrm{kg}$ and chloride concentration of $24 \mathrm{mmol} / \mathrm{kg}$ with a weight of sweat of $136 \mathrm{mg}$.

Two weeks before diagnosis the child's condition deteriorated, with increasing respiratory problems, weight loss, and watery diarrhoea. Once again $H$ influenzae was grown from sputum along with $C$ albicans. Serum tests for Pneumocystis carinii were negative. Once again she responded to antibiotic treatment guided by culture and sensitivity report.

The results of stool examination were negative for cryptosporidium, Isospora belli, and atypical mycobacteria.

A preliminary report from the immunology department that the child's T4 lymphocytes were reduced greatly, along with the hyperglobulinaemia and neutropenia, prompted us to check the patient's blood for antibody to HIV. This was positive on ELISA (enzyme linked immunosorbent assay) testing and confirmed by Western blot technique. These tests were repeated and found to be consistent.

At that point treatment with high dose intravenous co-trimoxazole and zidovudine by mouth was started. Intravenous human immunoglobulin was given and she was discharged from hospital to continue that treatment. During the first two months after discharge the child remained in better health, gaining weight and having less diarrhoea and respiratory symptoms. Her peripheral blood white cell count dropped and zidovudine was stopped and the co-trimoxazole changed to another antibiotic, but after a month her condition deteriorated with further respiratory infections and weight loss.

She was restarted on zidovudine and at the time of writing was receiving immunoglobulin intravenously once every three weeks. Her condition was deteriorating, however, and she was being fed through a nasogastric tube.

Her 2 year old sister, who was also IIIV positive, had three episodes of pneumonia over the past year, $H$ influenzae being isolated from gastric aspirate on the latest occasion. She also suffered weight loss, hyperglobulinaemia, and anaemia. Her sweat electrolyte concentrations were unequivocally normal. One other sibling, aged 9 months, and both parents were also HIV positive, but all were completely asymptomatic and had normal sweat test results.

\section{Discussion}

Many children present with frequent respiratory illnesses, and in the ones who are failing to thrive and who may also have diarrhoea a sweat test is usually performed to exclude or confirm cystic fibrosis. When the procedure is correctly performed it is generally accepted that a sweat sodium concentration of greater than $70 \mathrm{mmol} / \mathrm{kg}$ combined with that clinical picture suggests a diagnosis of cystic fibrosis. Other conditions, such as adrenal insufficiency, hypothyroidism, and diabetes insipidus, may be associated with abnormal sweat electrolyte concentrations, but our patient showed none of the clinical or biochemical features of any of these.

The use of fludrocortisone to suppress sweat electrolyte concentration was described in 1966, when Lobeck and McSherry observed that sweat sodium and chloride concentrations of patients with cystic fibrosis did not decrease if they were given large doses of $9 \alpha$-fludrocortisone. ${ }^{1}$ In our child there was a clear response to this test. 
Although $H$ influenzae may be found in the sputum of children with cystic fibrosis, we were surprised that Staphylococcus aureus was not also isolated. Furthermore, $C$ albicans is an unusual finding in children's sputum unless they have been receiving long term antibiotic treatment.

Diarrhoea and failure to thrive are common presenting features in children with symptomatic HIV infection. ${ }^{2}$ In our child the diarrhoea was not malabsorptive and did not respond to pancreatic replacement treatment. Organisms which may be suspected in this situation are protozoans such as cryptosporidium, microsporidia, and Isospora belli. A search should also be made for atypical mycobacteria, cytomegalovirus, and herpes simplex virus, any of which may cause a malabsorptive picture. Our patient had no evidence of any of these organisms or any evidence of neoplasia.

With the incidence of cystic fibrosis in the community of 1 in 2000 it would not be surprising that a child with clear evidence of cystic fibrosis responding to standard treatment should be found to be HIV positive (personal communication, Dr J Mok, consultant paediatrician, City Hospital, Edinburgh). Although initially HIV positive this child has since become HIV negative, therefore suggesting clearing of maternal HIV antibody.
Measuring immunoreactive trypsin is not helpful as a diagnostic aid beyond the neonatal period, and gene probe analysis would not have been useful as there was no family history of cystic fibrosis. We $\stackrel{\square}{2}$ think that there is little doubt that our patient had not got cystic fibrosis.

We therefore suggest that children presenting with symptoms suggestive of cystic fibrosis but with atypical features such as unexpected bacteriological results, failure to respond to treatment, \& or hyperglobulinaemia should have both their sweat test result m validated by a fludrocortisone suppression test and their HIV state checked. If the HIV test result is positive it will, as in our family, allow rationalisation of treatment and counselling for the family.

\section{References}

1 Lobeck CC, McSherry MS. Response of sweat electrolyte concentrations to 9 alphafluorohydrocortisone in patients with cystic fibrosis and their families. I Pediatr 1963;62:393-8. 2 Pahwa S, Kaplan M, Fikrig S, et al. Spectrum of human T cell lymphotropic virus type III in children. FAMA 1986;255:2299-305.

(Accepted 20 October 1987)

\section{SHORT REPORTS}

\section{Cervical adenocarcinoma and oral contraceptives}

Two recent papers from the United States reported a twofold to threefold increase in the incidence of invasive adenocarcinoma of the uterine cervix in women under the age of 35 between 1973 and $1982 .{ }^{12}$ This was not seen in older women or in squamous carcinoma of the cervix, which in the United
Cases were divided into adenocarcinoma (including adenosquamous carcinoma), 은 squamous cell carcinoma, and "unknown and unspecified" cancer. Non- $G$ epithelial tumours were omitted. Incidences were calculated in three age groups (20-34, 35-54, and 55 and over) and for three five year periods (1968-72, 1973-7, and 1978-82). Within each age group the incidences were standardised for age by the indirect method using five year age groups; the 1968-82 rates for South Thames were taken as standard.

The table shows that the incidence of adenocarcinoma of the cervix in women under the age of 35 increased from $2 \cdot 4$ to $6 \cdot 6 /$ million between $1968-72$ and 1978-82. The incidences of squamous and unclassified carcinomas also increased.

Standardised incidences of invasive cervical cancer (numbers of cases/million) by histological type and time period from three English regional cancer registries

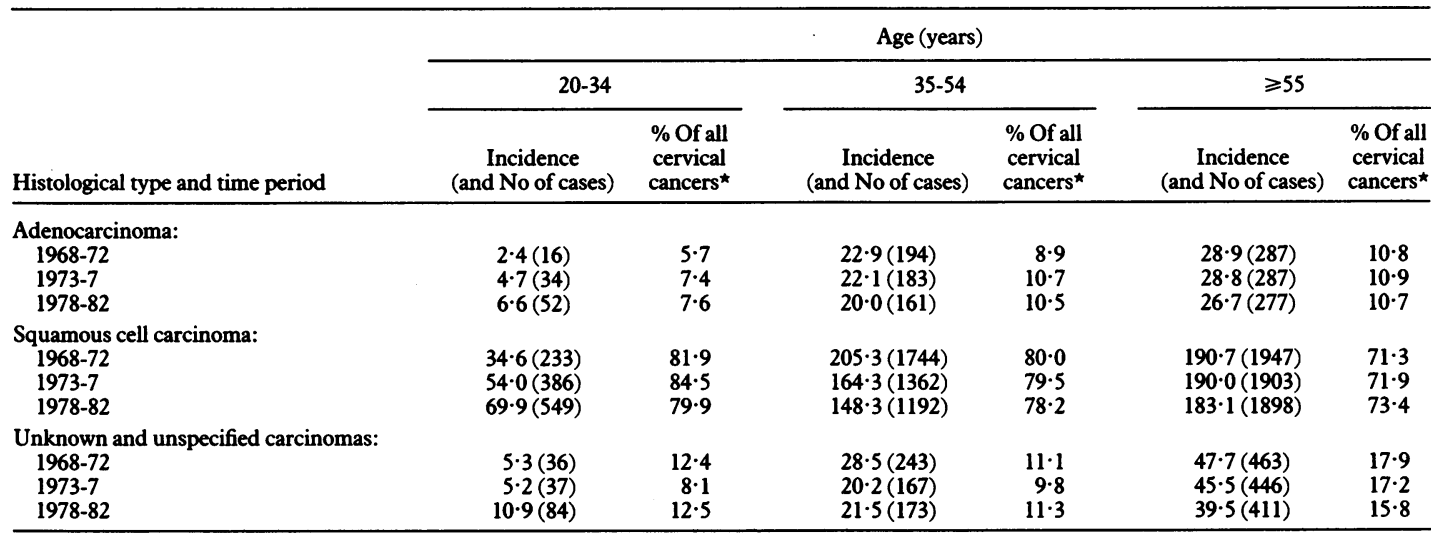

*Based on incidences.

States has been decreasing in women at all ages. Peters et al suggested that the increase in the incidence of adenocarcinoma might reflect the use of oral contraceptives by young women. ${ }^{1}$ Cervical changes, particularly "microglandular hyperplasia," within the endocervix of women with a history of oral contraceptive or hormone use have been reported, ${ }^{3}$ and DallenbachHellweg reported on a series of 23 women with invasive adenocarcinoma of the cervix in whom the prevalence of oral contraceptive use was unusually high. ${ }^{4}$ We report comparable English data on the incidence of cervical cancer.

\section{Methods and results}

Annual numbers of registrations of cervical cancer from 1968 to 1982 were obtained from the South Thames, Oxford, and West Midlands cancer registries.
Adenocarcinoma as a proportion of all cervical cancers increased from $5 \cdot 7 \%$ to $7 \cdot 6 \%$ in this age group.

In women aged 35-54 the incidences of both squamous carcinoma and adenocarcinoma declined, although the proportion of cervical adenocarcinomas rose slightly from $8.9 \%$ to $10.5 \%$. In women aged over 55 the incidence of both squamous carcinoma and adenocarcinoma declined slightly, and the relative proportion of each histological type changed little.

\section{Comment}

As in the United States, there has been a pronounced increase in the incidence of adenocarcinoma in young English women, which cannot be explained by more accurate classification of previously unspecified cancer. In England squamous cell cancer has also increased but to a lesser extent.

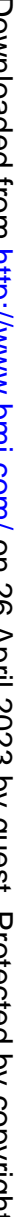

